\title{
Assessment of the relationship between gametocyte density, multiplicity of infection and mosquito infectivity in Plasmodium falciparum infections
}

\author{
Abdoulie Touray \\ PAUSTI \\ Victor Atunga Mobegi ( $\nabla$ vmobegi@gmail.com ) \\ University of Nairobi https://orcid.org/0000-0002-1962-5583 \\ Fred Wamunyokoli \\ Jomo Kenyatta University of Agriculture and Technology \\ Hellen Butungi \\ International Centre for Insect Physiology and Ecology \\ Jeremy K. Herren \\ International Centre for Insect Physiology and Ecology
}

\section{Research}

Keywords: P. falciparum, asymptomatic, gametocyte density, MOI, mosquito infection rates, Mbita

Posted Date: June 9th, 2020

DOI: https://doi.org/10.21203/rs.3.rs-33602/v1

License: (c) (1) This work is licensed under a Creative Commons Attribution 4.0 International License.

Read Full License 


\title{
1 Assessment of the relationship between gametocyte density, 2 multiplicity of infection and mosquito infectivity in \\ 3 Plasmodium falciparum infections
}

4

\author{
Abdoulie O. Touray ${ }^{* 1,4}$, Victor A. Mobegi ${ }^{* 2}$, Fred Wamunyokoli ${ }^{3}$, Hellen Butungi, ${ }^{4,5}$, and \\ Jeremy K. Herren ${ }^{* 4}$
}

Address: ${ }^{1}$ Department of Molecular Biology and Biotechnology, Institute of Basic Sciences, Technology and Innovation, Pan African University (PAUSTI), Nairobi, Kenya, ${ }^{2}$ Department of Biochemistry, School of Medicine, University of Nairobi, Nairobi, Kenya, ${ }^{3}$ Department of Biochemistry, Jomo Kenyatta University of Agriculture and Technology (JKUAT), Nairobi, Kenya, ${ }^{4}$ International Centre of Insect Physiology and Ecology (icipe), Nairobi, Kenya and ${ }^{5}$ Wits Research Institute for Malaria, School of Pathology, Faculty of Health Sciences, University of the Witswatersrand, Johannesburg, South Africa

Email: Abdoulie O. Touray* - abdoulietouray79@gmail.com; Victor A. Mobegi* vatunga@uonbi.ac.ke; Fred Wamunyokoli - fwamunyokoli@jkuat.ac.ke; Hellen Butungi hbutungi@gmail.com; Jeremy K. Herren* - jherren@icipe.org

* Corresponding authors.

\section{Abstract}

Background: Malaria is a major public health threat in sub-Saharan Africa. Asymptomatic $P$. falciparum gametocyte carriers are potential infectious reservoirs for sustaining transmission in many malaria endemic regions. The aim of the study was to assess the prevalence of gametocyte carriage and some of its associated risk factors among asymptomatic schoolchildren (age 5-15 years) in Mbita, western Kenya and further analyse the association between gametocyte density, multiplicity of infection (MOI) and mosquito infectivity.

Methods: Rapid diagnostic test (RDT) was used to screen for P. falciparum parasite infection among asymptomatic schoolchildren (5-15 years old) residing in Mbita, Western Kenya and the results were further verified using microscopy. Participants positive for $P$. falciparum infection were further screened for gametocyte carriage and those positive were used to feed laboratory 
31 reared An. gambiae s.s. mosquitoes using membrane feeding method. Genomic DNA was

32 extracted from dry blood spots (DBS) samples and $P$. falciparum populations were genotyped

33 using 10 polymorphic microsatellite markers. Assessment of the association between MOI and

34 gametocyte density and mosquito infection rates was conducted.

35 Results: The prevalence of Plasmodium falciparum infection among the study population was

$3629.1 \%$. A significantly higher $P$. falciparum infection was found among the male gender $(n=764$,

$37 p$-value $<0.05)$ compared to the females $(n=657)$. Gametocyte prevalence among the study 38 population was $2 \%$. Children (5-9 years) have a higher risk of gametocyte carriage (Odd Ratios = $392.1[95 \% \mathrm{CI}=1.3-3.4], P=0.002)$. Our results indicate a significant and positive combined 40 effect of gametocyte density and MOI on mosquito infection rate $(\mathrm{R}=0.825, p<0.0001)$.

41 Conclusion: The study reports a relatively stable year-round gametocyte carriage among the 42 study population. This pattern of gametocyte carriage signals the role of schoolchildren in 43 maintaining malaria transmission in the study area. A strong positive correlation was found 44 between gametocyte density, multiplicity of infection and mosquito infectivity.

45 Keywords: P. falciparum, asymptomatic, gametocyte density, MOI, mosquito infection rates, $46 \quad$ Mbita

\section{Background}

48 The intensification of global and local malaria control measures have led to remarkable 49 reductions in disease burden in many regions including sub-Saharan Africa. The incidence of $P$. 50 falciparum clinical cases and prevalence have respectively declined by $40 \%$ and $50 \%$ within the

51 African continent between 2000 and 2015 [1]. However, recent data indicates this trend might be 52 reversing, an estimated 213 million malaria cases and 380,700 related deaths in the World Health 
53 Organisation (WHO) African Region between 2017 and 2018, an increase relative to previous

54 years [2]. Clearly, malaria continues to be a serious public health problem in the continent

55 threatening the lives of many people particularly children and pregnant women. In Kenya, like

56 many other African countries, P. falciparum is the dominant parasite species with about $70.2 \%$

57 of the population at risk of the disease [3]. Malaria is one of the leading causes of hospital

58 admissions and death in the country accounting for about $30 \%$ and $19 \%$ outpatient and inpatient

59 cases respectively, with an estimated inpatient death of 3-5\% [2, 4].

60 The Kenyan government through the implementation of a national strategic malaria control plan

61 and subsequently, the launching of the next iteration of its national malaria strategy (KMS)

62 2019-2023, has intensified its fight against the disease in a bid to attain a "malaria free

63 Kenya". This involved the introduction and scaling up of interventions such as long-lasting

64 insecticide net (LLIN), rapid diagnostic test (RDT), and artemisinin-based combination therapy

65 (ACT) [5, 6]. The implementation of these interventions have resulted to a decline in malaria

66 transmission in many parts of the country [7]. Nevertheless, the coastal part of the country and

67 areas along the shores of Lake Victoria continue to face high malaria transmission [8].

68 Malaria parasite transmission from humans to the mosquito vectors requires the presence of 69 infectious mature gametocytes in the peripheral blood of the human host $[9,10]$. Based on the 70 central role of gametocytes in propagating and sustaining malaria transmission, the prevalence of

71 gametocytes is largely used as a parameter of the disease transmission [11, 12]. Studies in 72 malaria endemic and high transmission areas have reported high asexual parasite and gametocyte 73 prevalence and densities in children relative to adults [13, 14]. In such high malaria transmission 74 settings, due to repeated parasite exposure, older children and adults develop immunity against 75 the parasite $[15,16]$. As a result, this category of people are most likely to experience 
asymptomatic infections harbouring gametocytes at microscopic and sub-microscopic densities thereby serving as efficient parasite reservoirs for sustaining malaria transmission $[12,14,17]$. Reports about high prevalence of asymptomatic infections and gametocyte densities in schoolchildren have be documented in some malaria endemic areas [17, 18]. Asymptomatic malaria infections in schoolchildren mostly remain undiagnosed and are not treated due to the lack of clinical manifestation. Therefore, this group of people are largely neglected by most of the currently implemented malaria interventions and control programs $[18,17]$. In addition, following the decline in malaria burden in many endemic areas, information on the prevalence of asymptomatic $P$. falciparum infections and gametocyte carriage in schoolchildren particularly in remote settings in sub-Saharan Africa remains patchy [19]. Since asymptomatic infections and prevalence of gametocyte carriage in schoolchildren may significantly hamper the attainment of malaria control and elimination goals in sub-Saharan Africa $[18,20]$, it will be important to further investigate dynamics and infectivity of asymptomatic carriers.

The presence of gametocytes in the peripheral blood of the human host does not necessarily translate into mosquito infectivity $[9,21]$. Some of the major factors that influence the successful transmission of $P$. falciparum gametocytes to the mosquito vectors include, human attractiveness and exposure to the mosquito vectors, host and vector immune responses, seasonality, gametocyte maturity and densities, and multiplicity of infection (MOI) [21, 22]. MOI is the number of distinct parasite clones concurrently infecting a host. The link between MOI and gametocytemia of $P$. falciparum is still not fully elucidated [21], however, studies have reported a positive association between $\mathrm{MOI}$ and gametocyte carriage [14, 23]. The presence of genetically diverse multiple $P$. falciparum clones is reported to increase the chances of some parasite clones to evade the host anti-parasite immune responses thereby promoting gametocyte 
99 development and persistence [14, 24]. Mosquito infection rates of $P$. falciparum are reported to 100 be positively associated with gametocyte density particularly at high gametocyte concentrations 101 [25]. However, at low gametocyte concentrations, a varying and less strong association is 102 reported [9]. The nature of the tri-partite interaction between gametocyte density, MOI and 103 mosquito infectivity remains poorly documented.

104 Two common characteristics of asymptomatic malaria infections in endemic settings are the 105 prevalence of varying levels of gametocyte carriage among different age categories due to anti106 parasite immunity and high rates of polyclonal infections [15, 26, 27]. In order to ultimately 107 eliminate malaria, interventions geared towards interrupting the disease transmission through 108 efficient and effective identification and treatment of both asymptomatic and symptomatic 109 carriers will be of immense importance [14, 15]. Understanding the association between 110 gametocyte density, MOI and mosquito infectivity will enhance proper identification and 111 targeted treatment of parasite reservoirs responsible for sustaining the ongoing malaria

112 transmission in the region [14]. Here, we report on the prevalence of gametocyte carriage and 113 some of its associated risk factors among asymptomatic schoolchildren (age 5-15 years) in 114 Mbita, western Kenya and further assesses the association between gametocyte density, MOI and 115 mosquito infectivity.

\section{Methods}

\section{Study Site}

118 The study was carried out in Mbita sub-county (Suba North), in the Homa Bay County of 119 Western Kenya. Study participants were recruited from primary schools within Mbita sub-county 120 (within $50 \mathrm{~km}$ radius of Mbita town). The sub-county is situated on the shores of Lake Victoria 
121 and located between latitudes $0^{\circ} 21^{\prime}$ and $0^{\circ} 32^{\prime}$ 'South and longitudes $34^{\circ} 04^{\prime}$ and $34^{\circ} 24^{\prime}$ East.

122 The area of the district is about $163.28 \mathrm{~km}^{2}$ with a population of 124,938 (Figure 1).

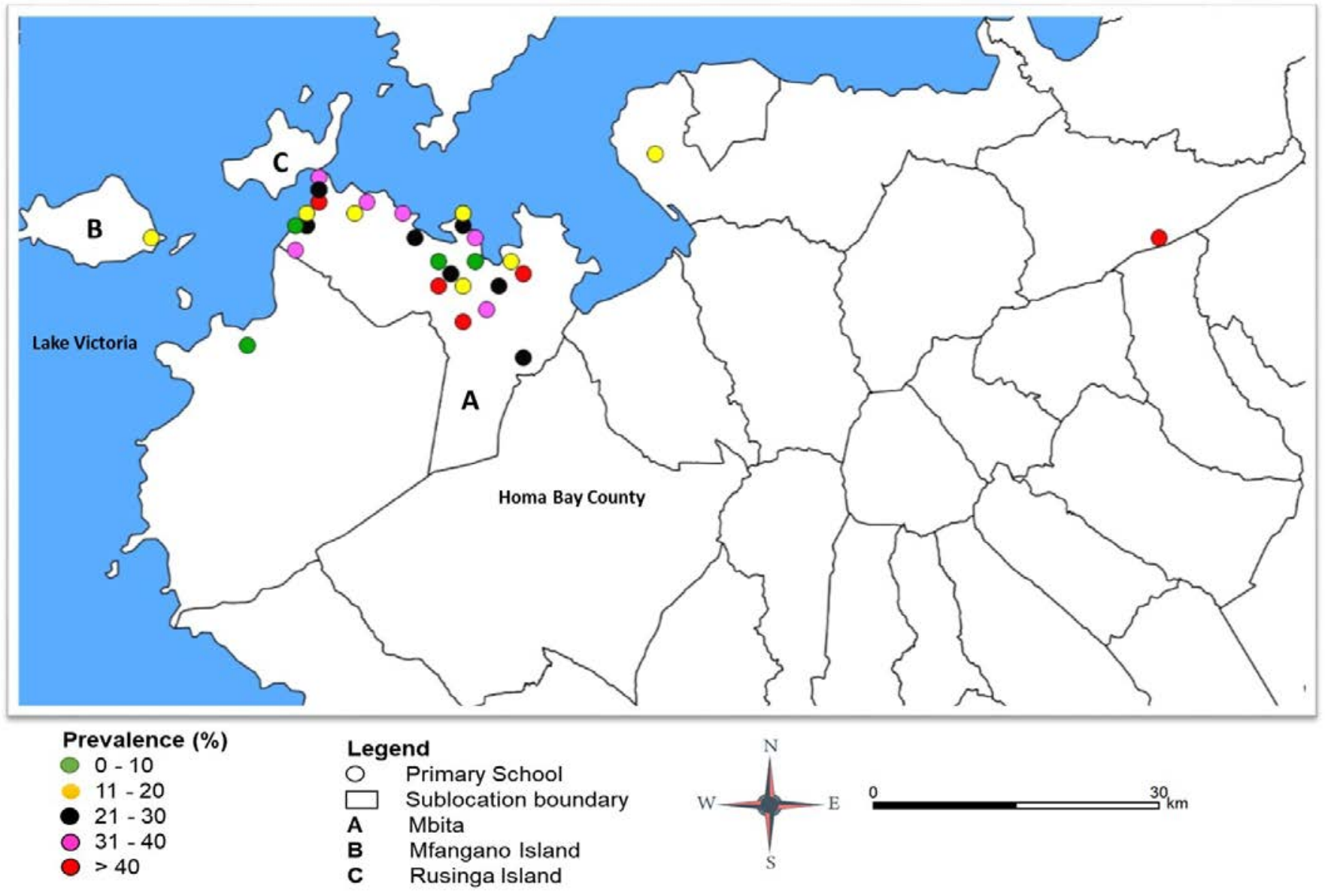

\section{Figure 1}

Map of Homa Bay County indicating the prevalence of Plasmodium falciparum infection among the schools in the study site. The site-specific prevalence (\%) was calculated as the percentage of $P$. falciparum positive infections within each school.

\section{Study subjects and Sample collection}

Primary school children between the ages 5 and 15 years residing in Mbita sub-county, Western

Kenya were recruited and screened for P. falciparum malaria infection using Rapid Diagnostic Test (RDT) (SD Bioline Malaria Ag P.f/Pan HRP-II/pLDH) and microscopy. Schoolchildren 
133 from the various primary schools around Mbita were enrolled in a study that commenced in

134 December 2016 to evaluate the effects of symbiotic microbes and mosquito vector competence.

135 Blood samples were collected from each participant for RDT and 10\% Giemsa stained thin and 136 thick blood films preparation for microscopy diagnosis of $P$. falciparum malaria infection and 137 also collected on a filter paper (Whatman 3MM; Whatman, Maidstone, United Kingdom) for 138 DNA extraction. The filter paper dried blood spots (DBS) were stored at $-20{ }^{\circ} \mathrm{C}$. An additional 4 $139 \mathrm{~mL}$ of venous blood was collected from participants with $P$. falciparum gametocytes as detected 140 by microscopy for use in the membrane feeding assays. Four thousand eight hundred eighty141 eight (4881) participants were screened in this study.

\section{Experimental infection of mosquitoes}

143 Venous blood samples $(4 \mathrm{~mL})$ collected from individuals who tested positive for P. falciparum 144 carriage using RDT and subsequently found to be gametocyte-positive from the microscopy 145 diagnosis, were immediately fed to the mosquitoes. Experimental feeds were carried out in 146 batches of 100 (per feeding cup) locally reared 3 to 5 day-old female An. gambiae s.s. 147 mosquitoes via an artificial membrane attached to a water-jacketed glass feeder maintained at 37 $148{ }^{\circ} \mathrm{C}$. A total of 37 gametocyte-positive venous blood samples collected from different individuals 149 was used to feed the mosquitoes. After 15-20 minutes, fully fed mosquitoes are selected and kept 150 on glucose for 7 days at $27^{\circ} \mathrm{C}-29^{\circ} \mathrm{C}$. On the $10^{\text {th }}$ day post-infection, the mosquitoes that were 151 alive were then collected and stored at $-20{ }^{\circ} \mathrm{C}$ in Eppendorf tubes. The proportion of infected 152 mosquitoes was determined by detecting the $P$. falciparum circumsporozoite protein (CSP) in the 153 stored mosquito samples using CSP ELISA as described elsewhere [28].

\section{Microsatellite genotyping}


155 Genomic DNA (gDNA) was extracted from the DBS samples using the QIAamp DNA Mini Kit 156 (51304, QIAGEN, Hilden, Germany) based on the manufacturer's protocol. gDNA quality and 157 concentration of each sample was determined using a Nanodrop 2000C (Thermo Fisher 158 Scientific, Waltham, MA, USA) and samples were stored at $-20{ }^{\circ} \mathrm{C}$ until used. The microsatellite 159 amplification, fragment analysis and MOI determination method used in this study is the same as 160 described by Mobegi et al. 2012 [29]. The samples analysed here are part of those used in our 161 previous study [30].

\section{Data storage and Analysis}

163 Age, gender, weight and Plasmodium parasiteamia of each study participant together with 164 mosquito infectivity and microsatellite genotyping data collected were carefully verified. 165 Descriptive statistics and Pearson Chi-Square test for significance between groups were 166 determined. Risk factors analysis was done using a binary logistic regression model and multiple 167 correlation and regression analysis was used to test for possible relationship between gametocyte 168 density, MOI and mosquito infection rates. Statistical analysis were conducted in IBM SPSS 169 Statistics for Windows, version 25 (IBM Corp., Armonk, N.Y., USA). Schools were mapped 170 using geographical information system (GIS) and the map generated using QGIS software 171 version 2.4.0.

\section{Results}

\section{Demographic and Parasitological characteristics of the study participants}

174 In this study, a total of 4881 schoolchildren (age 5-15 years) were screened using RDT and the 175 parasite status confirmed by microscopy. The total number of the female and male participants 176 were 2459 and 2422, respectively. Regarding the parasitological characteristics of the study 
177 participants, significant differences were observed among the male and female gender with

178 higher $P$. falciparum prevalence among the male gender $(764 / 1421, p$-value $<0.05)$. There was

179 no statistically significant difference in $P$. falciparum parasite carriage between the age groups

180 (5-9 and 10-15 years). The total number of mixed infections (P. falciparum plus $P$. ovale and/or

181 P. malariae) detected in the study population was 204 with a significantly higher prevalence

182 among the children between the ages 5-9 years $(112 / 204$, $p$-value $<0.05)$ while the single

183 infections were 1217. Most of the mixed infections were found among the female gender

$184(110 / 204, p$-value < 0.05) (Table 1 and Additional file 1).

185 Table 1: Parasitological characteristics of the study participants.

186

\begin{tabular}{cllll}
\hline Variables & \multicolumn{3}{c}{ RDT } & \\
\hline Age (years) & Positive & Negative & Total & $\chi^{2}(\boldsymbol{p}$-value $)$ \\
$5-9$ & 712 & 1833 & 2545 & \\
$10-15$ & 709 & 1627 & 2336 & $3.328(\mathbf{0 . 0 6 8})$ \\
Total & 1421 & 3460 & 4881 & \\
Gender & & & & \\
Female & 657 & 1802 & 2459 & \multirow{2}{*}{$13.770(<\mathbf{0 . 0 5})^{*}$} \\
Male & 764 & 1658 & 2422 & \\
Total & 1421 & 3460 & 4881 & $\chi^{2}(\boldsymbol{p}$-value) \\
\hline Age (years) & Mixed Infection & Single Infection & Total & \\
$5-9$ & 112 & 600 & 712 & \\
$10-15$ & 92 & 617 & 709 & \\
Total & 204 & 1217 & 1421 & \\
Gender & 110 & 547 & 657 & \\
Female & 94 & 670 & 764 & $5.661(<\mathbf{0 . 0 5})^{*}$ \\
Male & 204 & 1217 & 1421 & \\
Total & & MICROSCOPY & \\
\hline & Asexual & Gametocyte & Total & $\chi^{2}(\boldsymbol{p}$-value) \\
\hline Age (years) & & &
\end{tabular}

Page 9 of 24 


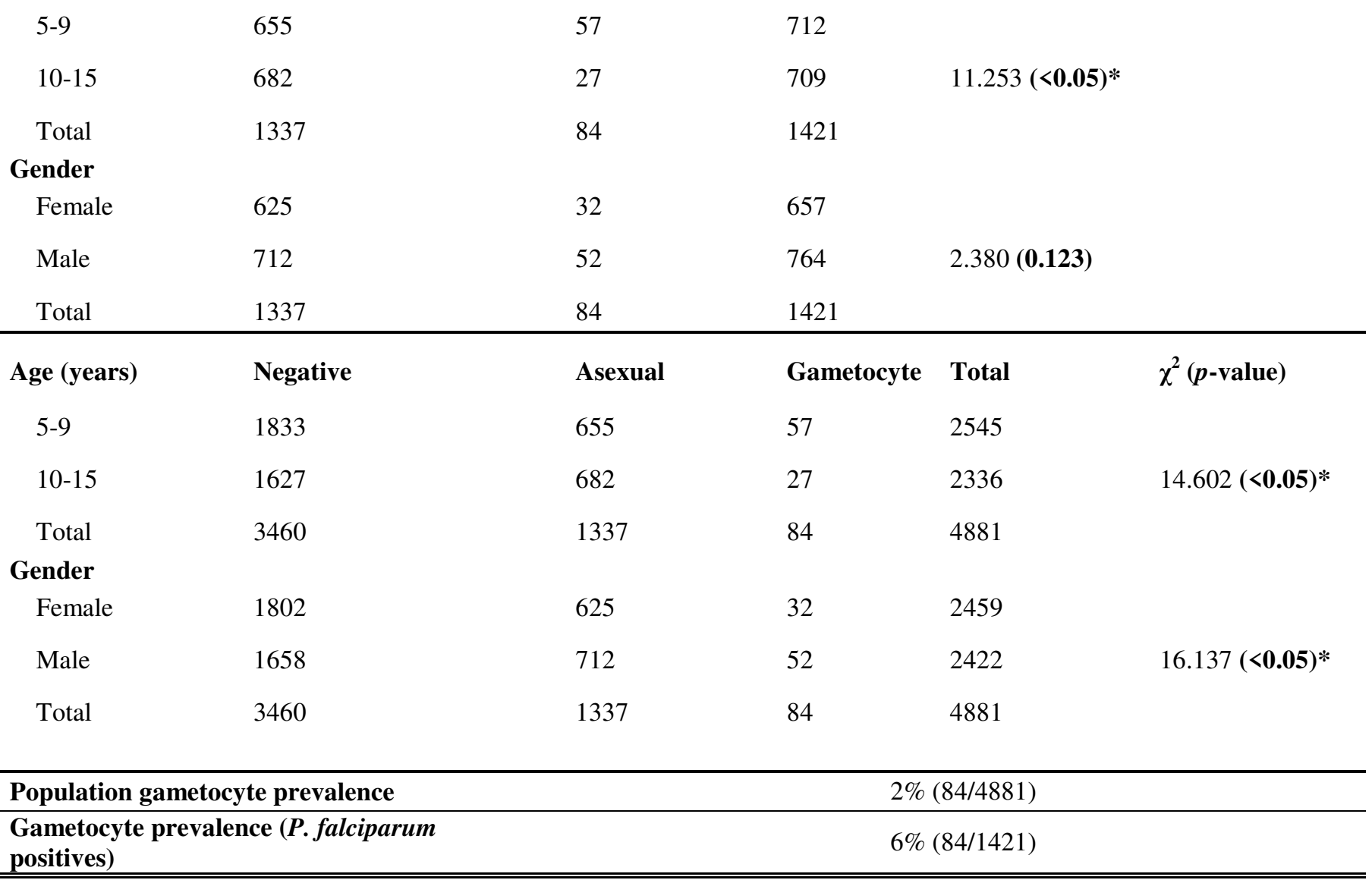

187

188

189

190

191

192

193 The population $P$. falciparum prevalence in this study calculated as the percentage of $P$.

194 falciparum infections within the study sample was $29.1 \%$. The level of $P$. falciparum carriage

195 varies among study sites (range: $0-65.2 \%, p$-value < 0.001) and within sampling periods (range:

196 11-78.4\%, $p$-value < 0.001) (Figure 1 and 2).

Population gametocyte prevalence is the percentage of gametocyte carriers among the total study population $(P$. falciparum positive and negative samples together) while the gametocyte prevalence among the $P$. falciparum positive samples is the percentage of gametocyte carriers among the $P$. falciparum positive samples only (excluding P. falciparum negatives). $\chi^{2}=$ Pearson's chi-squared test and $(*)$ indicates statistical significance.
Page 10 of 24 


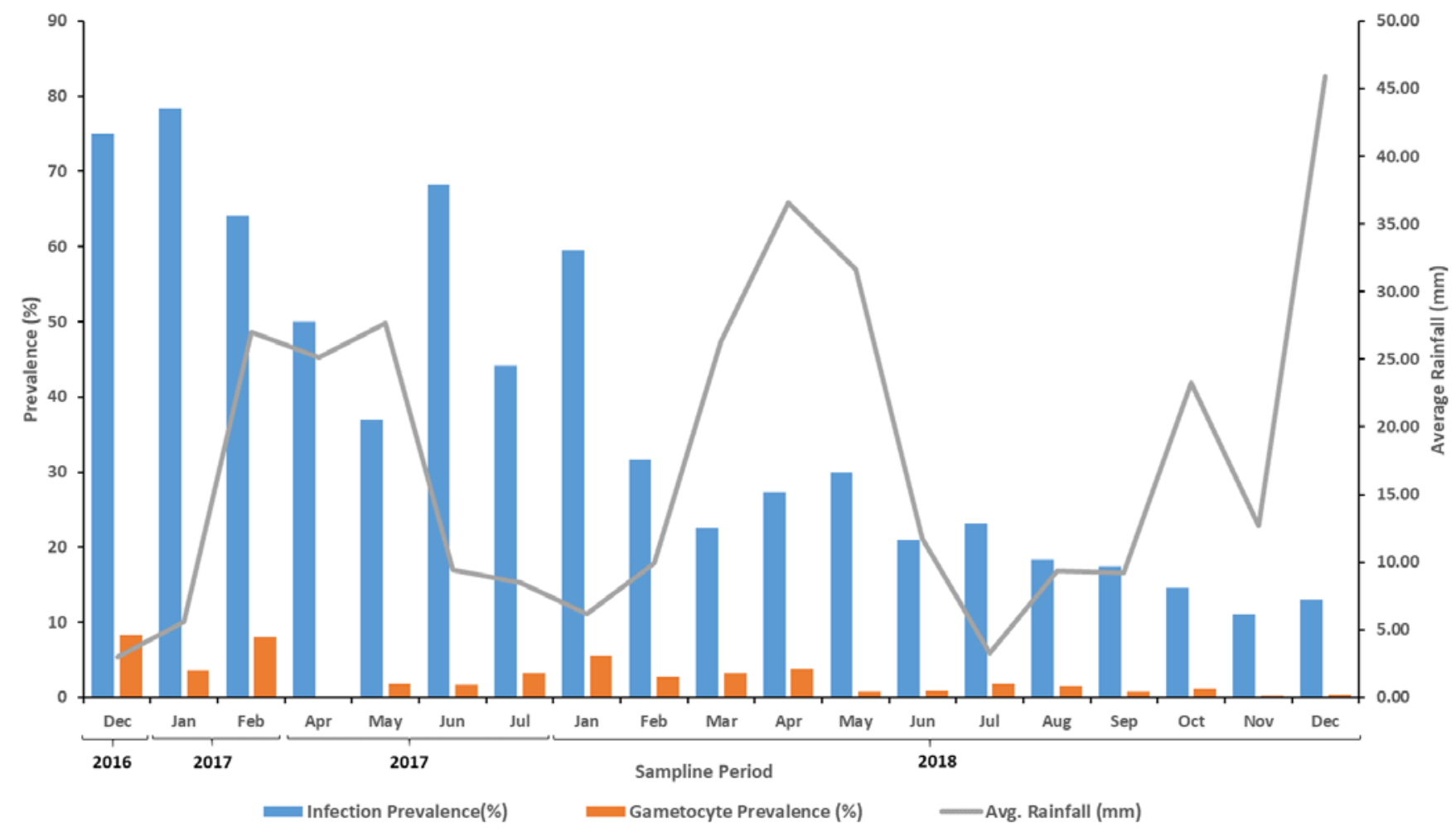

Figure 2

P. falciparum infection (blue) and gametocyte (brown) prevalence among the study participants and average rainfall (gray) during the various sampling periods.

201

\section{Prevalence of gametocyte carriage and associated risk factors in the study population}

In Table 1, the total number of gametocyte carriers was 84 with 57 of the carriers found within the age group 5-9 years as compared to 27 in the age-group $10-15$ years $(p$-value $<0.05)$. There was $2 \%$ population gametocyte prevalence in the study population. While the prevalence of gametocyte carriage among the $P$. falciparum malaria carriers (only $P$. falciparum positive

207 individuals) was found to be $6 \%$. These represent a minimum gametocyte prevalence level since 208 the sensitivity of microscopy is relatively low. There was significant difference in the population gametocyte carriage between the female (32) and male gender (52) ( $p$-value $<0.05)$. The 
gametocyte prevalence was relatively stable with no statistically significant difference

211 throughout the sampling periods (Figure 2).

212 The analysis showed that risk of $P$. falciparum infection was highest among the male gender as

213 compared to the females $(\mathrm{OR}=0.8[95 \% \mathrm{CI}=0.7-0.9], P<0.001)$ while the age of an

214 individual was not an independent risk factor. However, children between the ages of 5-9 years

215 have a higher risk of gametocyte carriage when infected with $P$. falciparum as compared to those

216 between the ages 10-15 years $(\mathrm{OR}=2.1[95 \% \mathrm{CI}=1.3-3.4], P=0.002)$ (Table 2$)$.

217 Table 2: Risk factors of $\boldsymbol{P}$. falciparum infection and gametocyte carriage.

\begin{tabular}{lcccccc}
\hline & \multicolumn{3}{c}{$\boldsymbol{P}$. falciparum parasite carriage } & \multicolumn{3}{c}{ Gametocyte carriage } \\
\hline Variable & OR & $\mathbf{9 5 \%}$ CI & P-value & OR & 95\% CI & $P$-value \\
Age (Years) & & & & & & \\
$5-9$ & 0.9 & $0.80,1.02$ & $\mathbf{0 . 1 0 1}$ & 2.14 & $1.34,3.43$ & $\mathbf{0 . 0 0 2 *}$ \\
$\quad 10-15$ & 1 & & & 1 & & \\
Gender & & & & 0.71 & $0.45,1.12$ & $\mathbf{0 . 1 3 6}$ \\
$\quad \begin{array}{l}\text { Female } \\
\text { Male }\end{array}$ & 0.79 & $0.70,0.90$ & $\mathbf{0 . 0 0 1 *}$ & 1 & & \\
\hline
\end{tabular}

Risk factors analysis of $P$. falciparum infection and gametocyte carriage among the study population using binary

223 The total number of samples used in assessing the relationship between gametocyte density and

224 MOI was 37. However, 15 of the 37 samples failed to amplify during the microsatellite 225 amplification PCR and are recorded as missing data. The mean mosquito infection rate was 226 12.71 (SE: 2.63, SD: 16.1) and mean gametocyte density was 59.89 (SE: 12.28, SD: 74.71), 227 respectively while the mean number of distinct alleles per isolate was 7.30 (SE: 0.80, SD: 3.76) 
228 (See Additional file 2). In this study, a significant positive correlation was found between $P$.

229 falciparum gametocyte densities in the patient blood samples and mosquito infection rates

$230(0.756, p$-value $<0.001)$. In addition, a positive correlation between multiplicity of $P$. falciparum

231 infection (MOI) and mosquito infection rates was reported $(0.451, p$-value $=0.018)$. However,

232 the correlation between MOI and gametocyte density was not statistically significant. The

233 mosquito infection rate is defined as the percentage of infected mosquitoes after day $10^{\text {th }}$ of the

234 membrane-feeding assay (Table 3 and Figure 3).

235 Table 3: Multiple correlation analysis of gametocyte density and multiplicity of $\boldsymbol{P}$. falciparum infection with 236 the infection rate in the mosquitoes.

237

\begin{tabular}{lccc}
\hline Parameters & Infection rate $(P$-value $)$ & Gametocyte density $(P$-value $)$ & MOI $(P$-value $)$ \\
\hline \hline Infection rate & $1($ Ref $)$ & $0.756(<\mathbf{0 . 0 0 1})^{*}$ & $0.451(\mathbf{0 . 0 1 8})^{*}$ \\
Gametocyte density & $0.756(<\mathbf{0 . 0 0 1})^{*}$ & $1($ Ref $)$ & $0.167(\mathbf{0 . 2 2 9})$ \\
MOI & $0.451(\mathbf{0 . 0 1 8})^{*}$ & $0.167(\mathbf{0 . 2 2 9})$ & $1($ Ref $)$ \\
\hline \hline
\end{tabular}

238 The dependent variable in this analysis is the infection rate. Ref represents the reference (*) denotes statistical 239 significance. 


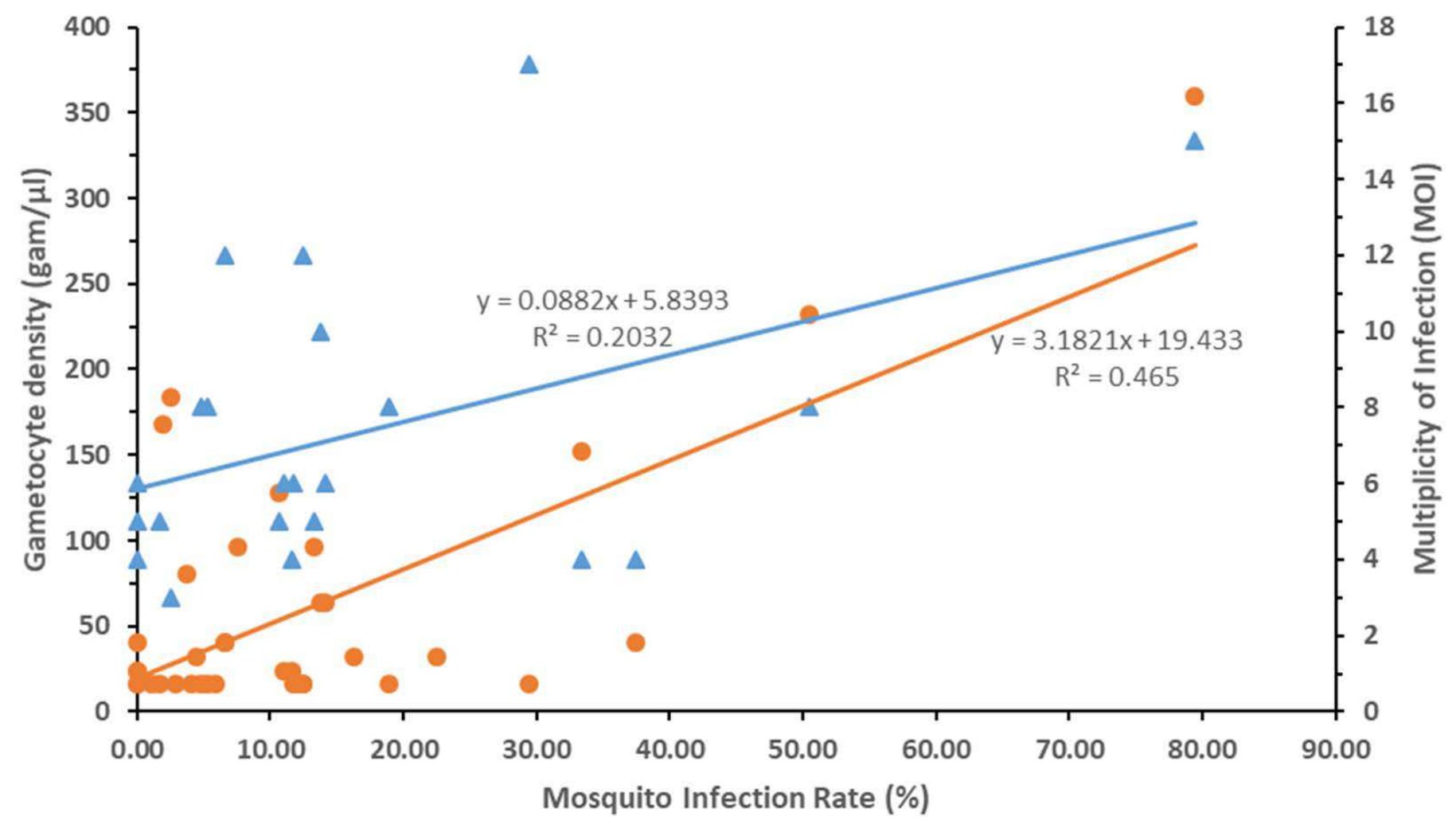

241

242 Figure 3

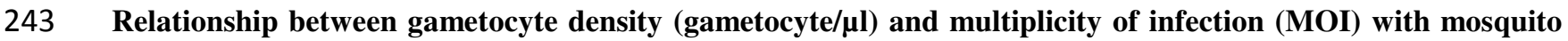
infection rate.

245

246 In Table 4, the multiple linear regression analysis showed that the collective effect of gametocyte

247 density and MOI has a strong positive correlation with mosquito infection rate $(\mathrm{R}=0.825$,

$248 \quad p<0.0001)$.

249 Table 4: Parameter of multiple linear Regressions Analysis.

\begin{tabular}{lllll}
\hline Parameters & Coefficients & Std. Error & t-statistic & $P$-value \\
\hline \hline (Constant) & -6.644 & 5.564 & -1.194 & $\mathbf{0 . 2 4 7}$ \\
Gametocyte density & 0.151 & 0.028 & 5.328 & $<\mathbf{0 . 0 1 0}$ \\
MOI & 1.707 & 0.672 & 2.54 & $\mathbf{0 . 0 2 0}$ \\
\hline \hline
\end{tabular}


$R=0.825, R-S Q R .=0.681, A d j . R-S Q R=0.647, S E=11.418 . R$ is the multiple correlation coefficient, $R-S Q R .(\mathrm{R}-$ square) is the multiple coefficient of determination, $A d j . R-S Q R$ represents the adjusted R-square, and $\boldsymbol{S E}$ is the standard error.

The multiple coefficient of determination $(\mathrm{R}-\mathrm{SQR} .=0.681)$ indicated that, about $68.1 \%$ of the variation in mosquito infection rate is accounted for by the gametocytes density and MOI. Thus, the formulated equation for mosquito infection rate in this study is:

$$
\hat{\mathrm{Y}}=-6.644+0.151 \mathrm{X}_{1}+1.707 \mathrm{X}_{2}
$$

Where $\hat{Y}$ is the expected Mosquito Infection rate, $\mathrm{X}_{1}$ and $\mathrm{X}_{2}$ are the Gametocytes density and MOI, respectively.

\section{Discussion}

This study assessed the prevalence of gametocyte carriers and some of the associated risk factors among asymptomatic schoolchildren (age 5-15 years) residing in Mbita, Western Kenya. In addition, an assessment of the relationship between gametocyte density, MOI and mosquito infection rates was also carried out. In this study, gametocyte prevalence among the $P$. falciparum positive individuals was $6 \%(84 / 1421)$ while the population $P$. falciparum gametocyte prevalence was $2 \%(84 / 4881)$. This corroborates with the finding of another study in the region [31, 32]. The intensification of the fight against malaria in the region by the Kenyan government may be the contributing factor for this relatively low gametocyte carriage reported in our study [33]. However, the changes in gametocyte prevalence in the study population needs further investigation using highly sensitive molecular tools in order to accurately estimate both the microscopic and submicroscopic gametocyte levels in the area. Gametocyte prevalence was higher among the younger age group (5-9 years) and accounted for 67.86\% (57/84) of the total 
273 gametocyte carriers in the study population. Similar pattern of gametocyte carriage was reported 274 by other studies [21, 31]. This can be explained by age-dependent development of anti-parasite 275 immunity due to repeated exposure in endemic settings [21, 31]. The high prevalence of 276 gametocyte carriage among the younger age group (5-9 years) pinpoints the potential role of this 277 age group in sustaining malaria transmission in the region. Children were reported to be 278 important contributors to the malaria infectious reservoir in many other settings [21]. Among the 279 P. falciparum malaria positive individuals, the male gender have higher proportion of both 280 asexual (764/1421) and gametocyte carriers (52/84) as compared to the females (asexual 281 carriage; 657/1421, gametocyte carriage; 32/84). However, the difference is not statistically 282 significant $(P$-value $=0.123)$. The $P$. falciparum prevalence was much lower in 2018 when 283 compared to the 2017 season. This is due to the 2018 indoor residual spraying (IRS) campaign 284 conducted by Africa Indoor Residual Spraying (AIRS) Kenya, in the region [34]. Nonetheless, 285 the gametocyte prevalence was relatively stable during all the sampling periods indicating a 286 year-round gametocyte carriage in the study population irrespective of the rainfall levels and 287 pattern. In malaria endemic settings, asymptomatic carriers are known to harbour gametocytes 288 even during the non-transmission season and are reported to be responsible for the resurgence of 289 malaria infections during the subsequent transmission season [32]. With a persistent relatively 290 high prevalence of mosquito vectors and asymptomatic $P$. falciparum gametocyte carriage, there 291 is a perennial transmission of malaria in the region with intense and relatively mild transmission 292 from April to August and November to January, respectively.

293 The only independent risk factor associated with $P$. falciparum infection found in this study was 294 gender. The male gender have higher odds of $P$. falciparum infection in the study area as 295 compared to the females. Gender was reported as a risk factor in other studies in the region [31]. 
296 This finding is in line with the reports that female children are biologically less susceptible to 297 infectious diseases as compared to the male children [35]. However, age was not found to be a 298 risk factor for contracting $P$. falciparum malaria infection in this study but was linked with 299 gametocyte carriage when infected with P. falciparum. Younger children (5-9 years) have higher 300 risk of gametocyte carriage when infected with P. falciparum. A study in Tanzania has also 301 reported similar association of age with increased gametocyte prevalence [36].

302 A positive correlation was found between gametocyte density and mosquito infection rates ( $p$ 303 value $<0.001)$. High infection rates were observed among mosquitoes that fed on carriers with 304 high gametocyte densities. This result corroborates with the findings of other studies [21, 37]. 305 However, Churcher et al. 2013 have reported a negative association between gametocyte density 306 and mosquito infection rates [38]. The relationship between multiplicity of P. falciparum 307 infection and mosquito infection rates is not well documented. This study reports a significant 308 correlation between MOI and mosquito infection rates ( $p$-value $=0.018)$. Gametocyte isolates 309 harbouring multiple distinct clones positively influence the mosquito infection rate. In contrast, a 310 negative association between MOI and mosquito infection prevalence and intensity was reported 311 elsewhere [39].

312 Gametocyte density is an important factor in predicting the success of $P$. falciparum transmission 313 to the mosquito vector. Nonetheless, other studies have stressed that gametocyte presence or 314 density alone in blood samples does not equal their infectiousness to mosquitoes [38]. Therefore, 315 understanding the relationship between the combined effects of gametocyte density and other 316 gametocyte factors like MOI with mosquito infection rate will improve our understanding of the 317 dynamics of $P$. falciparum transmission. Our results indicate a significant and positive combined 
318 effect of gametocyte density and multiplicity of $P$. falciparum infection on mosquito infection

319 rate. The results showed that about $68.1 \%$ of the variations in mosquito infection rate is

320 accounted for by the combined effect of MOI and gametocyte density. These results can be

321 explained by the emergence of highly virulent and infectious parasite strains due to intense intra-

322 host competition and high recombination rates among the distinct infecting clones [40, 41].

323 However, Morlais et al. 2015 reported a non-significant interaction of the combined effect of

324 MOI and gametocyte density on mosquito infection rate [39]. This difference may be due to the

325 fewer number of microsatellite markers (6 markers) used in their study as compared to 10

326 microsatellite markers in our study. In addition, the difference in mosquito species (An. coluzzii)

327 used in their studies might be another contributing factor since An. gambiae was reported to have

328 a higher blood-feeding rate compared to An. Coluzzii [42]. This finding will help in assessing the

329 infectious reservoirs in different malaria endemic regions thereby guiding the implementation of

330 targeted malaria control interventions.

331 The results presented here represents minimum level of gametocyte carriage, as some of the

332 microscopic and submicroscopic gametocytes might have been undetected.

\section{Conclusions}

334 Malaria prevalence and gametocyte carriage is high among the asymptomatic schoolchildren

335 particularly the younger age group (5-9 years) in the region. The relatively stable and year-round

336 prevalence of gametocyte carriage among the study participants in this study signals the role of

337 schoolchildren in maintaining malaria transmission in the study area. The strong positive

338 correlation between the combined effect of gametocyte density and MOI with mosquito infection

339 rates will help in determining the human infectious reservoirs in different malaria endemic 
340 settings. Therefore, this will help in identifying and treatment of asymptomatic malaria parasite

341 carriers in the community. Malaria control interventions that are targeted towards asymptomatic

342 gametocyte carriers and reduction in multiple clone parasite carriage could aid in the ultimate

343 elimination of the disease in the region.

\section{Ethics approval and consent to participate}

345 Parents or guardians of the children signed an informed consent form. The Kenya Medical 346 Research Institute (KEMRI) Scientific and Ethics Review Unit (SERU) granted approval for the 347 original study (KEMRI/RES/7/3/1). All experiments were performed in accordance with the 348 relevant guidelines and regulations.

\section{Consent for publication}

350 Not applicable

\section{Availability of data and materials}

352 The datasets used and analysed during the current study are available from the corresponding 353 author on reasonable request.

\section{Competing interests}

355 The authors declare that they have no competing interests.

\section{$356 \quad$ Funding}

357 This study was funded by Pan African University, Institute of Basic Sciences, Technology and 358 Innovation (PAUSTI) under the postgraduate training program awarded to AOT.

359 Authors' contributions 
AOT participated in data collection, analysis, interpretation and manuscript preparation. HB participated in data collection and manuscript preparation. VAM, FW and JKH participated in data interpretation and manuscript preparation. All authors read and approved the final manuscript.

\section{Acknowledgement}

We are indebted to all volunteers who partook in this study as well as their parents/guardians for granting consent. The authors are very much grateful to the entire research team of Dr. Jeremy K. Herren and the management of International Centre of Insect Physiology and Ecology (ICIPE) for permitting us to use their samples and giving us access to use their research facilities to conduct the study. Special thanks to Azumah Karim for his valuable assistance and advice in analysing the data.

\section{References}

1. Bhatt S, Weiss DJ, Cameron E, Bisanzio D, Mappin B, Dalrymple U, et al. The effect of malaria control on Plasmodium falciparum in Africa between 2000 and 2015. Nature. 2015;526:207-11.

2. World Health Organization. WHO. World Malar. Rep. 2019.

3. Kenya National Bureau of Statistics and ICF Macro. Kenya Demographic and Health Survey 2008-2009. Kenya National Bureau of Statistics and ICF Macro. 2010. http://apps.who.int/medicinedocs/documents/s17116e/s17116e.pdf\%0Ahttps://dhsprogra m.com/pubs/pdf/FR260/FR260.pdf

4. Division of Malaria Control,Kenya Ministry of Public Health and Sanitation. Malaria Control Strategy. 2009.

5. Division of Malaria Control, Kenya Ministry of Public Health K. The National Malaria Strategy 2001-2010. 2001.

6. Kenya Ministry of Health. Kenya Malaria Indicator Survey 2015. Minist. Heal. Kenya. 2016.

7. Macharia PM, Giorgi E, Noor AM, Waqo E, Kiptui R, Okiro EA, et al. Spatio-temporal 
analysis of Plasmodium falciparum prevalence to understand the past and chart the future of malaria control in Kenya. Malar J. 2018;17:1-13. https://doi.org/10.1186/s12936-018-

8. Noor AM, Gething PW, Alegana VA, Patil AP, Hay SI, Muchiri E, et al. The risks of malaria infection in Kenya in 2009. BMC Infect Dis. 2009;9:1-14.

9. Ouédraogo AL, Guelbéogo WM, Cohuet A, Morlais I, King JG, Gonçalves BP, et al. A 393 protocol for membrane feeding assays to determine the infectiousness of $P$. falciparum naturally infected individuals to Anopheles gambiae. MWJ. 2013;4:17-20.

10. Baker DA. Malaria gametocytogenesis. Mol Biochem Parasitol. Elsevier B.V.; 2010;172:57-65. http://dx.doi.org/10.1016/j.molbiopara.2010.03.019

11. Wampfler R, Timinao L, Beck HP, Soulama I, Tiono AB, Siba P, et al. Novel genotyping tools for investigating transmission dynamics of Plasmodium falciparum. J Infect Dis. 2014;210:1188-97.

12. Zeynudin A, Degefa T, Zeynudin A, Zemene E, Emana D, Yewhalaw D. High Prevalence of Gametocyte Carriage among Individuals with Asymptomatic Malaria : Implications for Sustaining Malaria Control and Elimination Efforts in Ethiopia. Hum Parasit Dis. 2015;2016:17-25.

13. Bousema JT, Meutstege AM, Sauerwein RW, Gouagna LC, Githure JI, Drakeley CJ, et al. Plasmodium falciparum gametocyte carriage in asymptomatic children in western Kenya. Malar J. 2004;3:1-6.

14. Lamptey H, Ofori MF, Kusi KA, Adu B, Yeboa EO, Baafour EK, et al. The prevalence of submicroscopic Plasmodium falciparum gametocyte carriage and multiplicity of infection in children, pregnant women and adults in a low malaria transmission area in Southern Ghana. Malar J. 2018;17:1-12. https://doi.org/10.1186/s12936-018-2479-y

15. Lindblade KA, Steinhardt L, Samuels A, Kachur SP, Slutsker L. The silent threat: Asymptomatic parasitemia and malaria transmission. Expert Rev. 2013;11:623-39.

16. Stone WJR, Dantzler KW, Nilsson SK, Drakeley CJ, Marti M, Bousema T, et al. 
Naturally acquired immunity to sexual stage $P$. falciparum parasites. Parasitology. 2016;143:187-98.

17. Coalson JE, Walldorf JA, Cohee LM, Ismail MD, Mathanga D, Cordy RJ, et al. High prevalence of Plasmodium falciparum gametocyte infections in school-age children using molecular detection: patterns and predictors of risk from a cross-sectional study in southern Malawi. Malar J. 2016;15:1-17.

18. Cohee L, Laufer M. Tackling malaria transmission in sub-Saharan Africa. Lancet Glob Heal. 2018;6:e598-9.

19. Brooker S, Clarke S, Snow RW, Bundy DAP. Malaria in African schoolchildren: options for control. Trans R Soc Trop Med Hyg. 2008;102:304-5.

20. Kanwugu ON, Helegbe GK, Aryee PA, Abdul-Karim A, Anaba F, Ziblim Z, et al. Prevalence of Asymptomatic Malaria among Children in the Tamale Metropolis: How Does the PfHRP2 CareStart ${ }^{\mathrm{TM}}$ RDT Perform against Microscopy? J Trop Med. 2019;2019:7.

21. Bousema T, Drakeley C. Epidemiology and infectivity of Plasmodium falciparum and Plasmodium vivax gametocytes in relation to malaria control and elimination. Clin Microbiol Rev. 2011;24:377-410.

22. Nilsson SK, Childs LM, Buckee C, Marti M. Targeting Human Transmission Biology for Malaria Elimination. PLoS Pathog. 2015;11:1-17.

23. Nwakanma D, Kheir A, Sowa M, Dunyo S, Jawara M, Pinder M, et al. High gametocyte complexity and mosquito infectivity of Plasmodium falciparum in the Gambia. Int $\mathbf{J}$ Parasitol. 2008;38:219-27.

24. Nassir E, Abdel-Muhsin AMA, Suliaman S, Kenyon F, Kheir A, Geha H, et al. Impact of genetic complexity on longevity and gametocytogenesis of Plasmodium falciparum during the dry and transmission-free season of eastern Sudan. Int J Parasitol. 2005;35:49_ 55.

25. Schneider P, Bousema T, Omar S, Gouagna L, Sawa P, Schallig H, et al. 
(Sub)microscopic Plasmodium falciparum gametocytaemia in Kenyan children after treatment with sulphadoxine-pyrimethamine monotherapy or in combination with artesunate. Int J Parasitol. 2006;36:403-8.

26. Grignard L, Gonçalves BP, Early AM, Daniels RF, Tiono AB, Guelbéogo WM, et al. Transmission of molecularly undetectable circulating parasite clones leads to high infection complexity in mosquitoes post feeding. Int J Parasitol. 2018;48:671-7. https://doi.org/10.1016/j.ijpara.2018.02.005

27. Greenwood BM. Asymptomatic malaria infections - Do they matter? Parasitol Today. 1987;3:206-14.

28. Stone W, Grabias B, Lanke K, Zheng H, Locke E, Diallo D, et al. A comparison of Plasmodium falciparum circumsporozoite protein-based slot blot and ELISA immunoassays for oocyst detection in mosquito homogenates. Malar J. 2015;14:1-9.

29. Mobegi VA, Loua KM, Ahouidi AD, Satoguina J, Nwakanma DC, Amambua-Ngwa A, et al. Population genetic structure of Plasmodum falciparum across a region of diverse endemicity in West Africa. Malar J. 2012;11:1-9.

30. Touray AO, Mobegi VA, Wamunyokoli F, Herren JK. Diversity and Multiplicity of $P$. falciparum infections among asymptomatic school children in Mbita, Western Kenya. Sci Rep. 2020;10:1-8. http://dx.doi.org/10.1038/s41598-020-62819-w

31. Omondi P, Burugu M, Matoke-Muhia D, Too E, Nambati EA, Chege W, et al. Gametocyte clearance in children, from western Kenya, with uncomplicated Plasmodium falciparum malaria after artemether-lumefantrine or dihydroartemisinin-piperaquine treatment. Malar J. 2019;18:1-9. https://doi.org/10.1186/s12936-019-3032-3

32. Idris ZM, Chan CW, Kongere J, Gitaka J, Logedi J, Omar A, et al. High and Heterogeneous Prevalence of Asymptomatic and Sub-microscopic Malaria Infections on Islands in Lake Victoria, Kenya. Sci Rep. 2016;6:1-13.

33. Muthui MK, Mogeni P, Mwai K, Nyundo C, Macharia A, Williams TN, et al. Gametocyte carriage in an era of changing malaria epidemiology: A 19-year analysis of a malaria longitudinal cohort. Wellcome Open Res. 2019;4:1-31. 
34. Kenya End of Spray Report 2018. Rockville MD. The PMI Africa IRS (AIRS) Project, Abt Associates Inc. 2018.

35. Gadalla AAH, Schneider P, Churcher TS, Nassir E, Abdel-Muhsin AMA, RanfordCartwright LC, et al. Associations between season and gametocyte dynamics in chronic Plasmodium falciparum infections. PLoS One. 2016;11:1-15.

36. Nyarko SH, Cobblah A. Sociodemographic determinants of malaria among under-five children in Ghana. Malar Res Treat. 2014;2014.

37. Akim NIJ, Drakeley C, Kingo T, Simon B, Senkoro K, Sauerwein RW. Dynamics of $P$. falciparum gametocytemia in symptomatic patients in an area of intense perennial transmission in Tanzania. Am J Trop Med Hyg. 2000;63:199-203.

38. Churcher TS, Bousema T, Walker M, Drakeley C, Schneider P, Ouédraogo AL, et al. Predicting mosquito infection from Plasmodium falciparum gametocyte density and estimating the reservoir of infection. elife. 2013;2:1-12.

39. Morlais I, Nsango SE, Toussile W, Abate L, Annan Z, Tchioffo MT, et al. Plasmodium falciparum mating patterns and mosquito infectivity of natural isolates of gametocytes. PLoS One. 2015;10:1-14.

40. Alizon S, de Roode JC, Michalakis Y. Multiple infections and the evolution of virulence. Ecol Lett. 2013;16:556-67.

41. Wargo AR, De Roode JC, Huijben S, Drew DR, Read AF. Transmission stage investment of malaria parasites in response to in-host competition. Proc R Soc B Biol Sci. 2007;274:2629-38.

42. Akogbéto MC, Salako AS, Dagnon F, Aïkpon R, Kouletio M, Sovi A, et al. Blood feeding behaviour comparison and contribution of Anopheles coluzzii and Anopheles gambiae, two sibling species living in sympatry, to malaria transmission in Alibori and Donga region, northern Benin, West Africa. Malar J. 2018;17:1-12. https://doi.org/10.1186/s12936-018-2452-9 
Figures

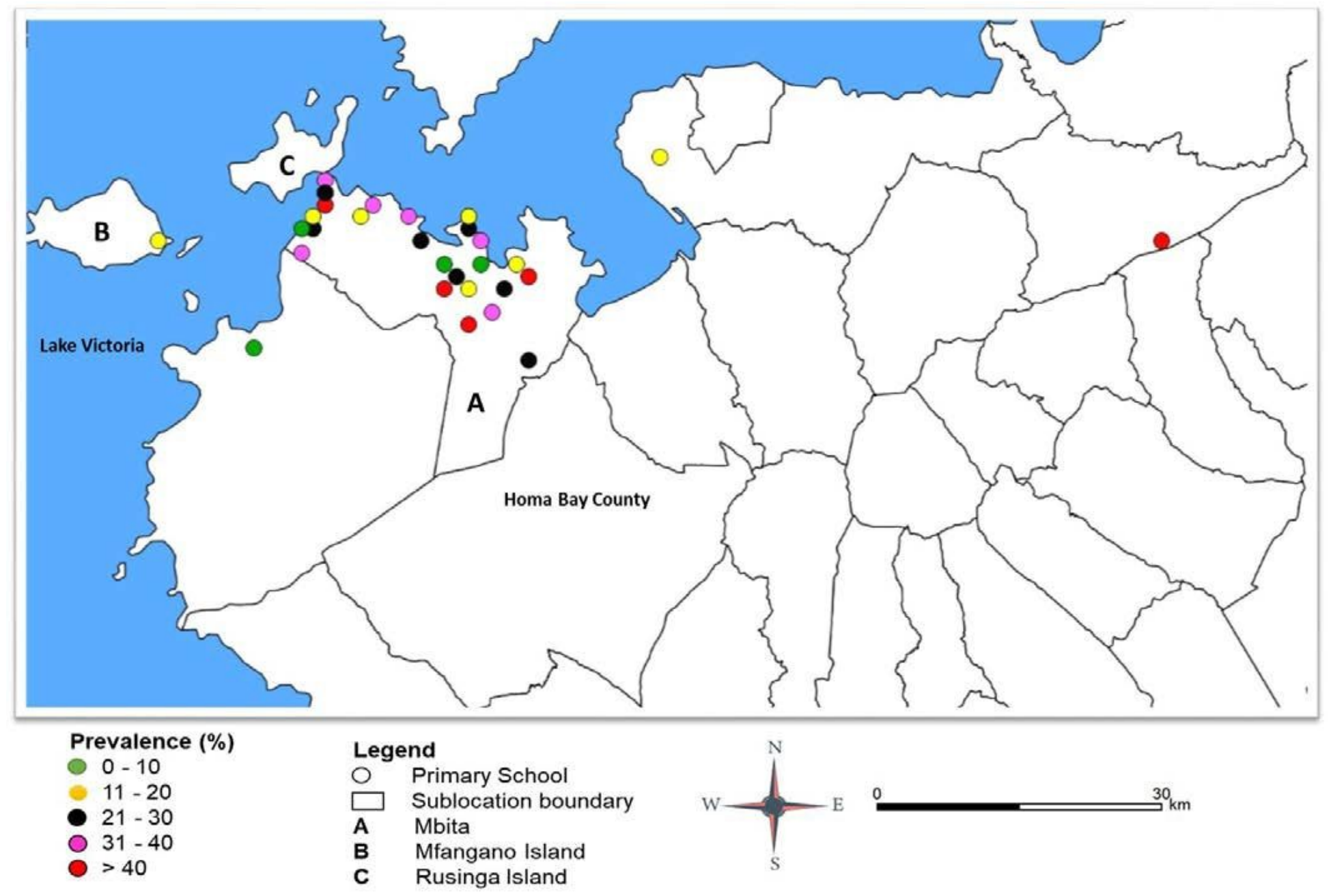

\section{Figure 1}

Map of Homa Bay County indicating the prevalence of Plasmodium falciparum infection among the schools in the study site. The site-specific prevalence (\%) was calculated as the percentage of P. falciparum positive infections within each school. 


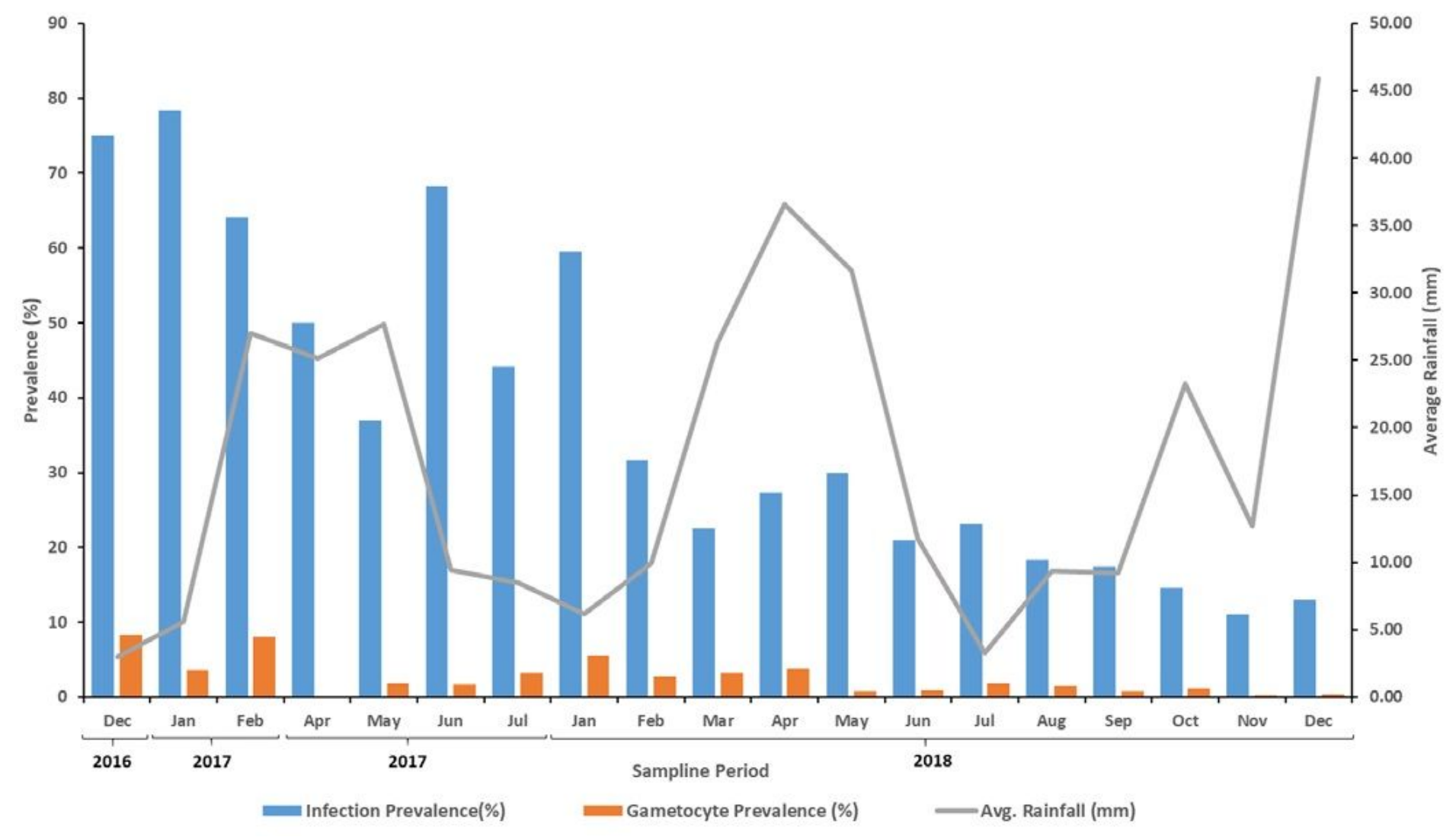

Figure 2

P. falciparum infection (blue) and gametocyte (brown) prevalence among the study participants and average rainfall (gray) during the various sampling periods. 

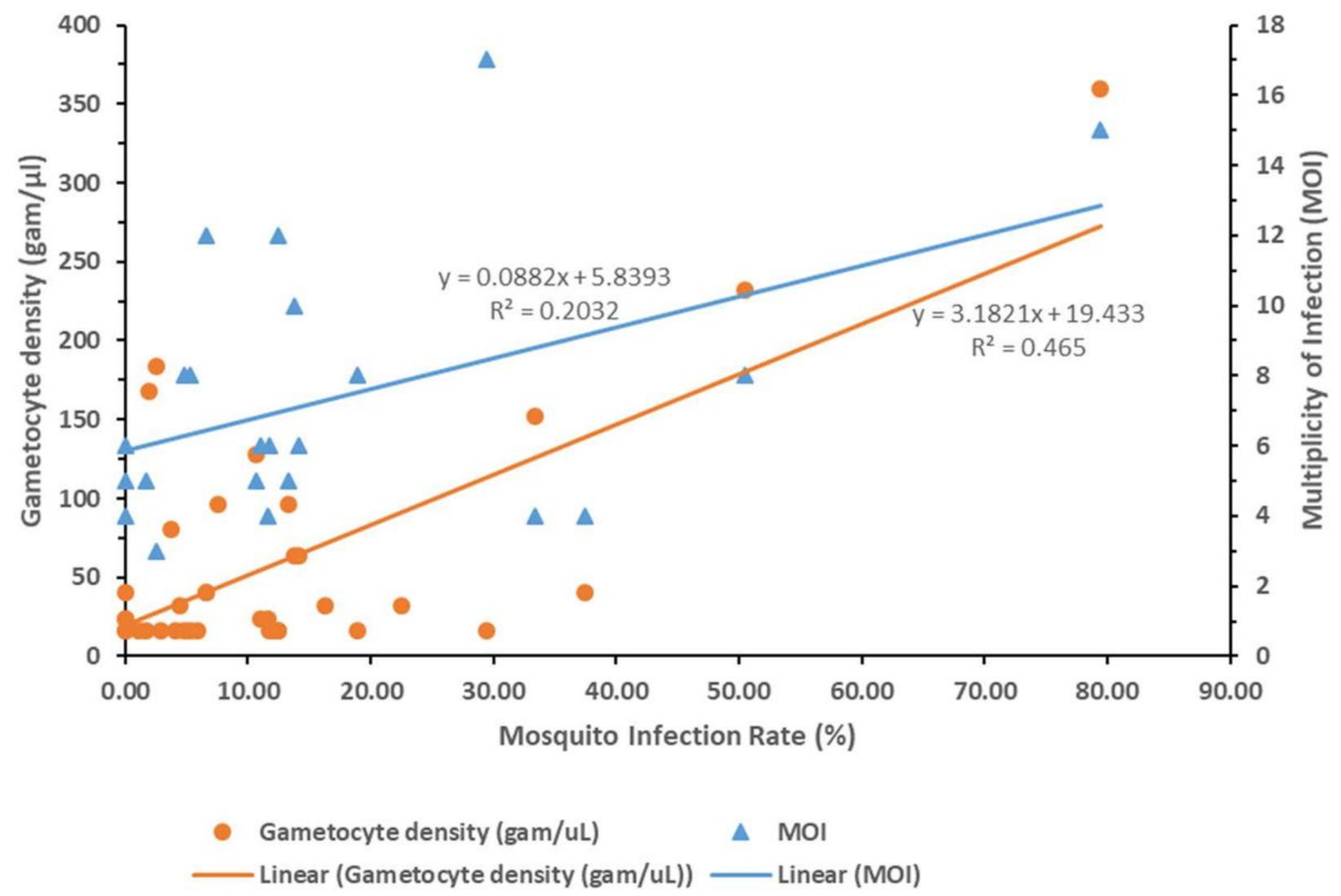

Figure 3

Relationship between gametocyte density (gametocyte/ $\mu \mathrm{l}$ ) and multiplicity of infection (MOI) with mosquito infection rate.

\section{Supplementary Files}

This is a list of supplementary files associated with this preprint. Click to download.

- Additionalfile1.docx

- Additionalfile2.docx 